\title{
Computed tomography and magnetic resonance imaging findings of malignant fibrous histiocytoma of the head and neck
}

\author{
JING LI ${ }^{1,2^{*}}$, ZHI-JUN GENG ${ }^{1,2^{*}}$, XIAO-FEI LV ${ }^{1,2}$, XIN-KE ZHANG ${ }^{1,3}$ and CHUAN-MIAO XIE ${ }^{1,2}$ \\ ${ }^{1}$ Collaborative Innovation Center for Cancer Medicine, State Key Laboratory of Oncology in South China; Departments of \\ ${ }^{2}$ Medical Imaging and ${ }^{3}$ Pathology, Cancer Center, Sun Yat-Sen University, Guangzhou, Guangdong 510060, P.R. China
}

Received August 3, 2015; Accepted February 25, 2016

DOI: $10.3892 / \mathrm{mco} .2016 .811$

\begin{abstract}
The aim of this study was to analyze the imaging findings of 15 cases of malignant fibrous histiocytoma (MFH) of the head and neck, in order to better understand the tumor characteristics based on computed tomography (CT) and magnetic resonance imaging (MRI). CT (n=11) and MRI $(n=4)$ images from 15 patients with histologically diagnosed MFH of the head and neck were retrospectively analyzed. The imaging characteristics were analyzed and compared among different histological subtypes. The lesions were primarily located in the maxillary sinus in 5 patients, the ethmoid sinus in 1 patient, the infratemporal fossa in 2 patients, the neck in 4 patients, the left mandible in 1 patient, the gingiva in 1 patient and the epiglottis in 1 patient. A total of 4 cases were associated with radiotherapy. All the lesions were ill-defined, ranging in size from 2.1 to $5.1 \mathrm{~cm}$ in the largest diameter. Bone fractures were evident in 9 cases. The attenuation, signal and enhancement patterns varied, although inflammatory MFH exhibited prolonged enhancement in dual-phase enhanced CT scans. MFH of the head and neck is rare but may be associated with radiotherapy. Although the imaging manifestations of MFH are various and non-specific, inflammatory MFH exhibits prolonged enhancement on dual-phase enhanced CT scans.
\end{abstract}

\section{Introduction}

Malignant fibrous histiocytoma (MFH) was first described by Ozzelo et al (1) and by O'Brien and Stout (2). MFH lesions are composed of different proportions of differentiated fibroblasts and histiocytes arranged in a storiform pattern (2).

Correspondence to: Dr Chuan-Miao Xie or Dr Xiao-Fei Lv, Department of Medical Imaging, Cancer Center, Sun Yat-Sen University, 651 Dongfeng Road East, Guangzhou, Guangdong 510060, P.R. China

E-mail: xiechuanmiao@yeah.net

E-mail: lvxf@sysucc.org.cn

*Contributed equally

Key words: malignant fibrous histiocytoma, magnetic resonance imaging, computed tomography
MFH is the most common soft tissue sarcoma in adults and is generally considered a high-grade sarcoma with poor prognosis $(3,4)$. MFH may occur at a wide range of ages, ranging mainly from 20 to 70 years. MFHs most commonly occur in the lower extremities and the retroperitoneum, whereas they are relatively uncommon in the head and neck, accounting for 3-10\% of all MFHs (5). Thus far, there have been only a limited number of case reports on MFHs in the head and neck, and only a few studies have included a description of the radiological findings (6-9). Due to its rare occurrence, there are few reports focusing on the computed tomography (CT) or magnetic resonance imaging (MRI) findings of MFH of the head and neck. Park et al (10) first reported the imaging characteristics of 13 patients with histologically diagnosed MFH of the head and neck, although the availability of information on the imaging characteristics of MFH remains limited. The purpose of our study was to present the CT and MR imaging characteristics of 15 cases of MFH arising in the head and neck.

\section{Patients and methods}

Patients. This retrospective study was approved by the Institutional Review Board of Sun Yat-Sen University (Guangzhou, China). Data were retrospectively collected from the medical records of patients with MFH histologically diagnosed at the Sun Yat-sen University Cancer Center between 2006 and 2013. The study included a total of 15 patients ( 9 men and 6 women), ranging in age from 20 to 77 years, with a mean age of 50 years. Among these patients, 4 developed MFH following radiotherapy for nasopharyngeal carcinoma or chondrosarcoma; the remaining patients were diagnosed with primary MFH. In 6 cases, the tumor recurred between 5 months and 2 years after surgery. All the MRI and CT images were reviewed and analyzed.

Imaging techniques. MRI scans were performed in 4 patients and CT scans in 11 patients. MR imaging was performed using a 1.5T system (Signa Excite II 1.5; GE Medical Systems, Waukesha, WI, USA) or a 3.0T system (Trio Tim; Siemens Medical Solutions, Erlangen, Germany) with a head and neck combined coil. Non-contrast-enhanced T1-weighted images $(\mathrm{TR}=500-600 \mathrm{msec}, \mathrm{TE}=10-20 \mathrm{~ms})$ in the axial, coronal and sagittal planes, and non-contrast-enhanced T2-weighted 
images $(\mathrm{TR}=4,000-6,000 \mathrm{msec}, \mathrm{TE}=95-110 \mathrm{msec})$ in the axial plane were obtained. Following intravenous injection of gadopentetate dimeglumine at a dose of $0.1 \mathrm{mmol} / \mathrm{kg}$ body weight, T1-weighted $(\mathrm{TR}=320-350 \mathrm{msec}, \mathrm{TE}=10-20 \mathrm{msec})$ fat-suppressed axial, coronal and sagittal sequences were obtained sequentially, with a 5-mm section thickness and 1-mm intersection gaps.

CT scans were obtained with a Brilliance TM16 (Philips Medical Systems, Best, The Netherlands) or a Toshiba Aquillion TM64 (Toshiba Medical Systems, Otawara, Japan) helical CT system. The main imaging parameters were as follows: $120 \mathrm{kV}, 250-300 \mathrm{~mA}$; section thickness, $5 \mathrm{~mm}$; field of view, $25 \mathrm{~cm}$; and matrix, 512x512. An intravenous bolus dose of $90 \mathrm{ml}$ non-ionic iodinated contrast agent (iopromide; Ultravist, Schering, Berlin, Germany) was administered at a rate of $3 \mathrm{ml} / \mathrm{sec}$.

Two experienced radiologists reviewed all the CT and MRI images and analyzed the imaging characteristics, including tumor location, size, margin, internal architecture, density or signal intensity and enhancement pattern.

\section{Results}

Tumor characteristics. Of the 15 MFHs in the head and neck in our series, 5 cases were located in the maxillary sinus, 1 in the ethmoid sinus, 2 in the infratemporal fossa, 4 in the neck, 1 in the left mandible, 1 in the gingiva and 1 in the epiglottis.

There were 7 lesions with histological subtypes that were classifiable, including 4 inflammatory, 2 storiform-pleomophic and 1 undifferentiated. Of the 4 inflammatory types, 1 case had undergone an MRI scan, and 3 cases had undergone CT scans. One case of the storiform-pleomophic type had undergone CT scan at first presentation and a subsequent MRI scan when the tumor recurred.

Imaging findings. The CT/MRI imaging findings of the 15 MFHs are presented in the Table I. All the lesions appeared as ill-defined, ranging in size from 2.1 to $5.1 \mathrm{~cm}$ in the largest diameter. Six sinonasal lesions, 2 infratemporal fossa lesions and 1 mandibular lesion were accompanied by bone fracture. On unenhanced CT images, the lesions $(n=11)$ were isoattenuated relative to the adjacent muscle; $2 / 11$ lesions were homogeneous and 9/11 lesions were heterogeneous, with areas of hypoattenuation. Calcifications were observed in 1 case that received irradiation treatment for primary chondrosarcoma. Following injection of contrast agent, 5 lesions exhibited heterogeneous mild-moderate enhancement, 5 exhibited heterogeneous marked enhancement, and 1 lesion exhibited ring-like marked enhancement. There were only 2 cases in which dual-phase enhanced CT scans were performed: One was an inflammatory MFH and exhibited markedly prolonged enhancement (Fig. 1); the subtype of the other could not be classified and exhibited mildly prolonged enhancement (Fig. 2).

On T1-weighted images, the lesions $(n=4)$ were isointense, with small foci of hyperintensity in 2 cases and heterogeneous isointensity in 2 cases. On T2-weighted images, all the lesions exhibited heterogeneous hyperintensity. One case exhibited small foci of short-T2 signals that were histologically identified as calcifications (Fig. 3). On post-contrast images, the lesions exhibited heterogeneous moderate-marked enhancement in
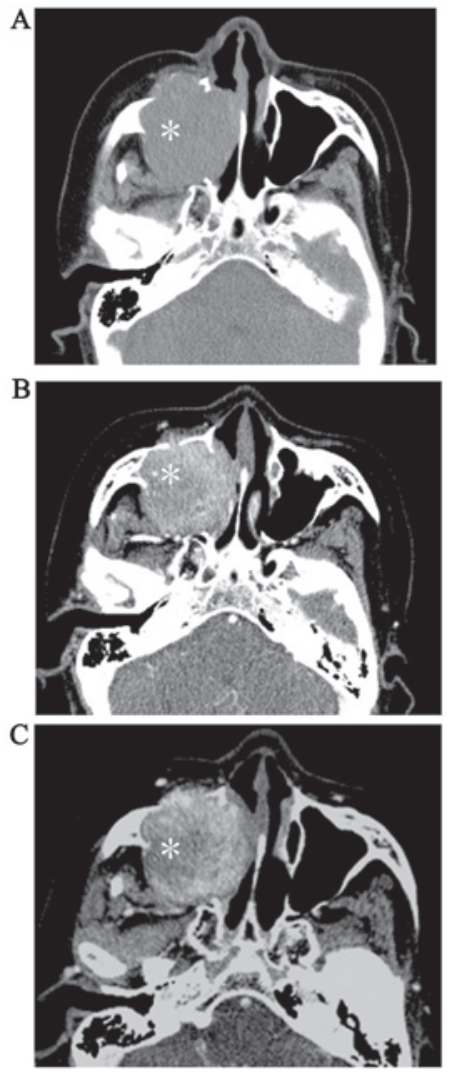

Figure 1. Inflammatory malignant fibrous histiocytoma of the right maxillary sinus (asterisks) in a 61-year-old woman. (A) Non-contrast computed tomography $(\mathrm{CT})$ image revealing a round, ill-defined homogeneous mass with bone fracture. (B) Contrast-enhanced CT image in the arterial phase exhibiting mild heterogeneous enhancement. (C) Contrast-enhanced CT image in the venous phase exhibiting markedly prolonged heterogeneous enhancement.

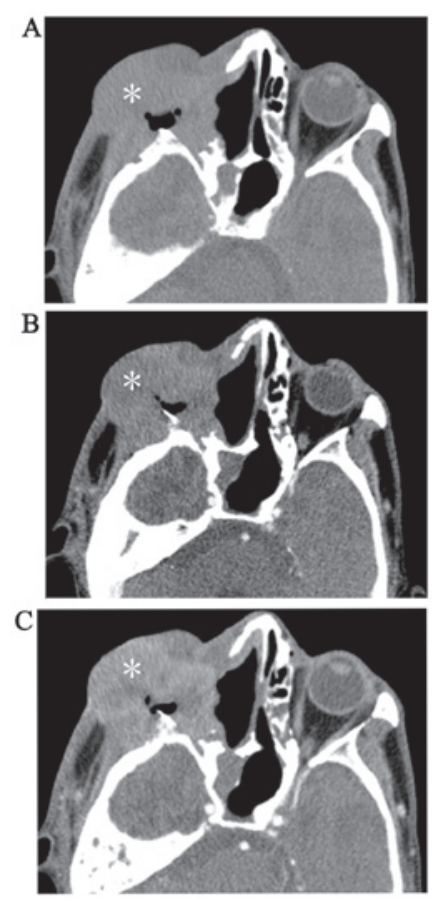

Figure 2. Recurrent malignant fibrous histiocytoma of the right orbit (asterisks) in a 43-year-old man. (A) Non-contrast computed tomography (CT) image revealing an irregular, ill-defined homogeneous mass in the right orbit. (B) Contrast-enhanced CT image in the arterial phase exhibiting mild heterogeneous enhancement. (C) Contrast-enhanced CT image in the venous phase exhibiting mild prolonged heterogeneous enhancement. 


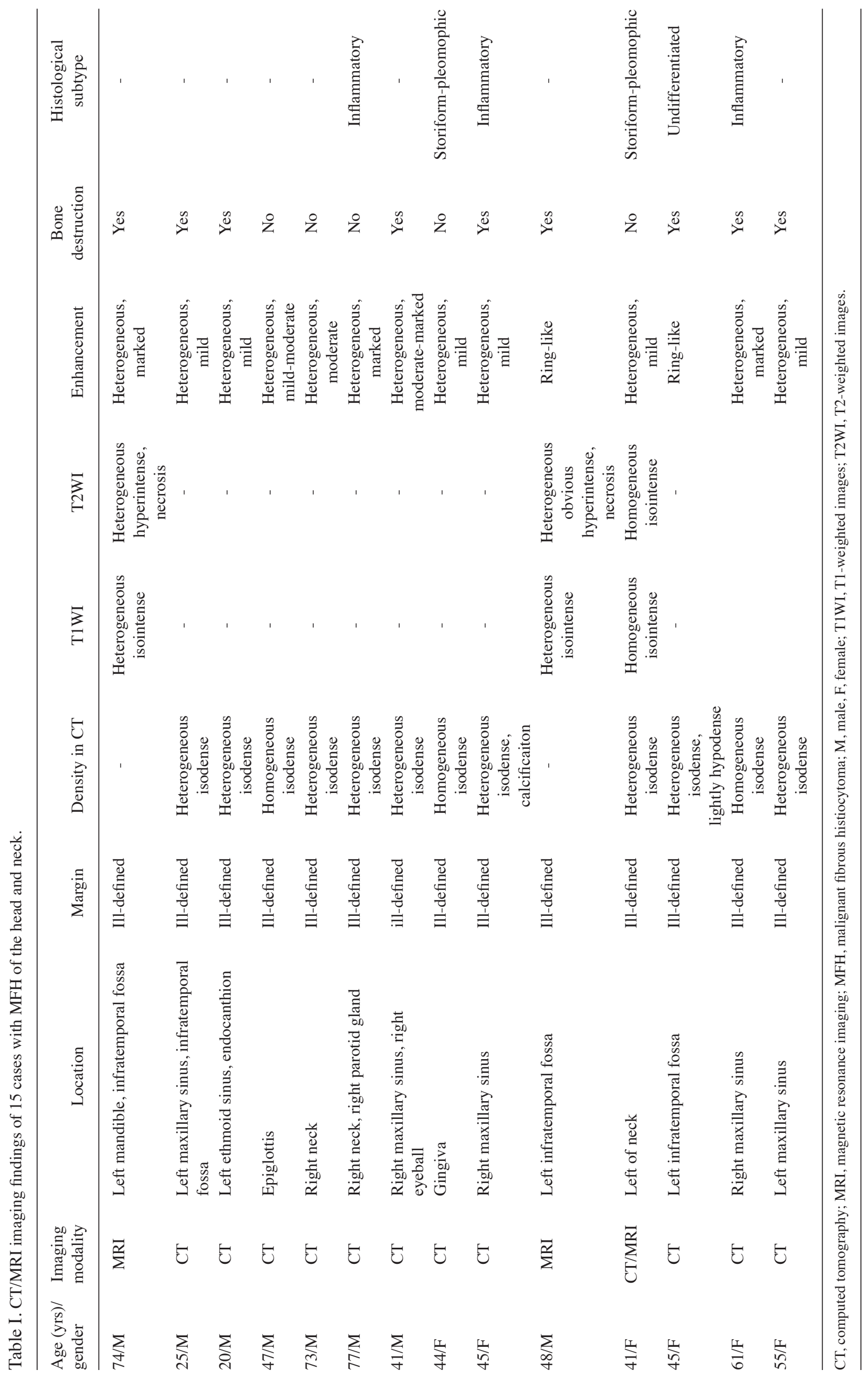



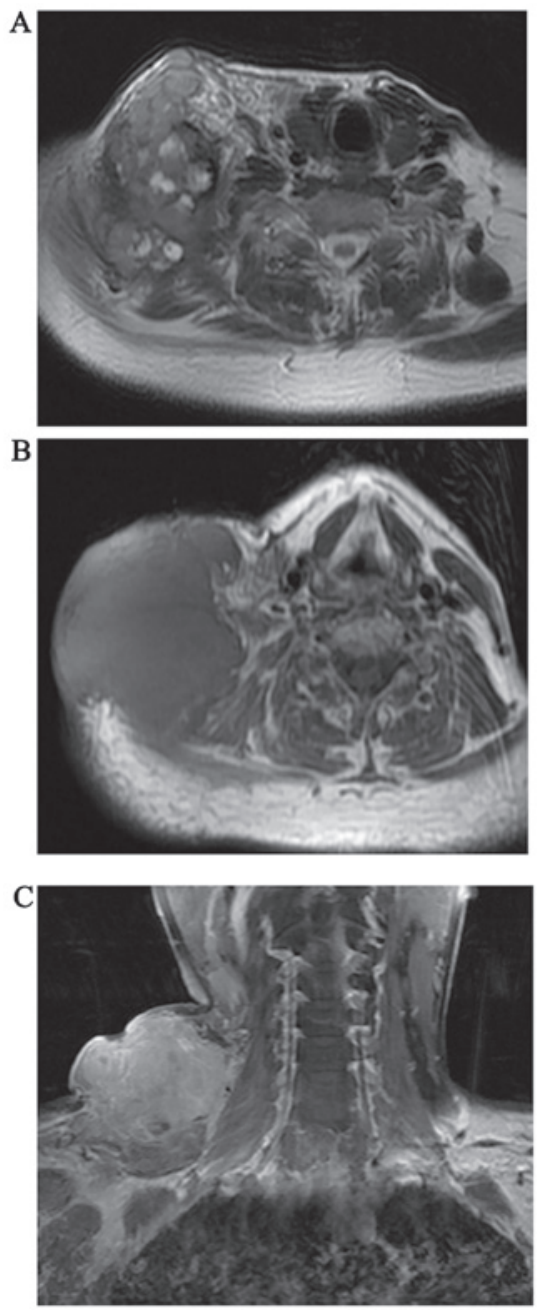

Figure 3. Inflammatory malignant fibrous histiocytoma of the neck in a 62-year-old man. (A) T2-weighted image revealing the small foci of a short-T2 signal. (B) T1-weighted image revealing a large, lobulated, round, hypointense mass. (C) Gadolinium-enhanced fat-suppressed T1-weighted coronal image revealing a mass with marked heterogeneous enhancement.

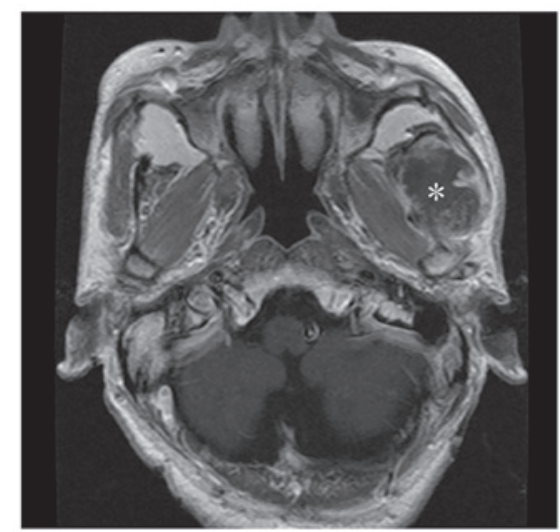

Figure 4. Gadolinium-enhanced fat-suppressed T1-weighted image revealing a mass with marked ring-like heterogeneous enhancement in the left infratemporal fossa (asterisk).

3 cases and ring-like marked enhancement in 1 case (Fig. 4). The non-enhancement areas represented necrosis in 2 ring-like enhancement lesions.

\section{Discussion}

MFH is a potentially devastating sarcoma that may occur at any age, although it is particularly prevalent in the fifth to seventh decades of life, with a male predominance. MFH frequently arises in the lower extremities, followed by the upper extremities and retroperitoneum (11). The incidence of MFHs in the head and neck is relatively low, accounting for only $3-10 \%$ of all MFHs according to the literature (5). The sinonasal tract has been reported to be the most common site of MFH involvement in this region $(12,13)$. In our series, the most common sites were the maxillary sinus (5/15), the neck $(4 / 15)$ and the infratemporal fossa $(2 / 15)$. MFH is reported to be one of the most common soft tissue sarcomas in adults; the majority of MFHs are high-grade lesions and frequently recur locally. Surgery is considered the primary treatment for $\mathrm{MFH}$ in the head and neck region, although the addition of chemotherapy and radiotherapy appears to be helpful for certain patients $(14,15)$. Lymphatic metastases are also rare, ranging between 0 and $15 \%$ (11). Only 1 case exhibited lymphatic metastasis in our series. All the cases underwent surgical excision; in 6 cases the tumor recurred, whereas the remaining cases were lost to follow-up.

$\mathrm{MFH}$ is reported to be the most common radiation-induced sarcoma (RIS) in the head and neck region (16). However, there are no studies demonstrating the association between specific radiation doses and RIS. Some researchers (17) have suggested that a total dose of $\geq 55$ Gy may increase the risk of RIS. Cai et al (18) described 59 cases of RIS in the head and neck, including 10 (16.9\%) MFHs, which ranked third in the series; the first and second most common types of RIS were fibrosarcoma and osteosarcoma, respectively. There were 4 cases of post-irradiation MFH in our series; the remaining cases were primary MFHs.

Clinically, patient symptoms often depend on the location of the tumor and its proximity to adjacent structures. Pain is the main symptom of MFH in the sinonasal tract due to the limited size of the sinus cavity; the sinus walls are always involved as the tumor grows. Patients may also present with nasal obstruction and epistaxis. $\mathrm{MFH}$ in the neck usually presents as a painless, enlarging mass.

MFH was first described in 1963, and the debate over the origin of the tumor cells has persisted for several years. MFH is currently considered to be synonymous with high-grade undifferentiated pleomorphic sarcoma. MFH was previously subdivided into five types: i) Storiform-pleomophic; ii) myxoid; iii) inflammatory; iv) giant-cell; and v) angiomatoid. However, according to the 2002 World Health Organization classification, the subtypes of $\mathrm{MFH}$ have been revised. For example, myxoid $\mathrm{MFH}$ and angiomatoid $\mathrm{MFH}$ are no longer considered to be subtypes of $\mathrm{MFH}$, whereas giant-cell MFH has been renamed undifferentiated pleomorphic sarcoma with giant cells, with the diagnosis made in the absence of differentiation. Inflammatory MFH was also renamed undifferentiated pleomorphic sarcoma with prominent inflammation, and this diagnosis is confirmed only if all the markers of mesenchymal lineage are negative. Storiform-pleomorphic MFH remains a category of fibrohistiocytic tumors $(19,20)$ and is the most common subtype in the head and neck region. 
MFHs lack typical CT/MRI imaging characteristics due to the variable degrees of histological differentiation. In particular, the density/signal intensity and enhancement pattern on CT/MRI are varied and exhibit no differences from other malignant soft tissue tumors. The tumor typically presents as a round mass with ill-defined margins and always invades the adjacent bones and soft tissue. The density/signal intensity is typically heterogeneous, although necrosis may be observed in the lesions, appearing as lightly hypodense/marked hyperintense regions on T2-weighted images without enhancement. Calcification or ossification may also be observed in $~ 5-20 \%$ of these lesions (10). In our series, necrosis was detected in 5 of the 15 cases. Calcification was detected on CT images in 1 case with prior osteosarcoma; the other case that underwent MRI also exhibited calcification in the pathological specimen, which appeared as a hypointense lesion on T2-weighted images. The tumors exhibited different degrees of heterogeneous enhancement on CT and MRI images. Park et al (10) analyzed 13 cases of MFH and reported that the signal and enhancement patterns of MFH were non-specific, with the exception of the myxoid type, which exhibited homogeneous marked hyperintensity on T2-weighted images and homogeneous marked enhancement. There were no myxoid-type MFH cases in our series; however, there were 4 cases of inflammatory MFH that exhibited marked enhancement, and 1 case exhibited prolonged enhancement in the venous phase. This enhancement pattern has not been previously reported and may suggest that inflammatory MFH is hypervascular. However, not all inflammatory MFHs exhibit marked enhancement. Karki et al (21) reported 3 cases of inflammatory MFH, although only 1 case exhibited marked enhancement, whereas the remaining 2 cases exhibited mild enhancement. These different enhancement patterns may be the result of different tumor locations.

In conclusion, the imaging findings of $\mathrm{MFH}$ are non-specific. However, inflammatory MFH of the head and neck may exhibit marked and prolonged enhancement. The diagnosis of MFH should be considered if the patient presents with a history of radiotherapy.

\section{References}

1. Ozzello L, Stout AP and Murray MR: Cultural characteristics of malignant histiocytomas and fibrous xanthomas. Cancer 16: 331-344, 1963.

2. O'Brien JE and Stout AP: Malignant fibrous xanthomas Cancer 17: 1445-1455, 1964.
3. Wanebo HJ, Koness RJ, MacFarlane JK, Eilber FR, Byers RM, Elias EG and Spiro RH: Head and neck sarcoma: Report of the Head and Neck Sarcoma Registry. Society of Head and Neck Surgeons Committee on Research. Head Neck 14: 1-7, 1992.

4. Potter BO and Sturgis EM: Sarcomas of the head and neck. Surg Oncol Clin N Am 12: 379-417, 2003.

5. Sturgis EM and Potter BO: Sarcomas of the head and neck region. Curr Opin Oncol 15: 239-252, 2003.

6. Yanagi Y, Murakami J, Hisatomi M, Katase N, Nagatsuka H and Asaumi J: A case of malignant fibrous histiocytoma of the maxillary sinus. Oral Surg Oral Med Oral Pathol Oral Radiol Endod 109: e99-e104, 2010.

7. Senel FC, Bektas D, Caylan R, Onder E and Gunhan O: Malignant fibrous histiocytoma of the mandible. Dentomaxillofac Radiol 35: 125-128, 2006

8. Nagano H, Deguchi K and Kurono Y: Malignant fibrous histiocytoma of the bucca: A case report. Auris Nasus Larynx 35: 165-169, 2008.

9. Satomi T, Watanabe M, Kaneko T, Matsubayashi J, Nagao T and Chiba H: Radiation-induced malignant fibrous histiocytoma of the maxilla. Odontology 99: 203-208, 2011.

10. Park SW, Kim HJ, Lee JH and Ko YH: Malignant fibrous histiocytoma of the head and neck: CT and MR imaging findings. AJNR Am J Neuroradiol 30: 71-76, 2009.

11. Weiss SW and Enzinger FM: Malignant fibrous histiocytoma: An analysis of 200 cases. Cancer 41: 2250-2266, 1978.

12. Sabesan T, Xuexi W, Yongfa Q, Pingzhang T and Ilankovan V: Malignant fibrous histiocytoma: Outcome of tumours in the head and neck compared with those in the trunk and extremities. Br J Oral Maxillofac Surg 44: 209-212, 2006.

13. Rodrigo JP, Fernández JA, Suárez C, Gómez J, Llorente JL and Herrero A: Malignant fibrous histiocytoma of the nasal cavity and paranasal sinuses. Am J Rhinol 14: 427-431, 2000.

14. Clark DW, Moore BA, Patel SR, Guadagnolo BA, Roberts DB and Sturgis EM: Malignant fibrous histiocytoma of the head and neck region. Head Neck 33: 303-308, 2011

15. Hardison SA, Davis PL III and Browne JD: Malignant fibrous histiocytoma of the head and neck: A case series. Am J Otolaryngol 34: 10-15, 2013.

16. Cakir O, Topal U, Bayram AS and Tolunay S: Sarcomas: Rare primary malignant tumors of the thorax. Diagn Interv Radiol 11: 23-27, 2005.

17. Patel SG, See AC, Williamson PA, Archer DJ and Evans PH: Radiation induced sarcoma of the head and neck. Head Neck 21: 346-354, 1999.

18. Cai PQ, Wu YP, Li L, Zhang R, Xie CM, Wu PH and Xu JH: $\mathrm{CT}$ and MRI of radiation-induced sarcomas of the head and neck following radiotherapy for nasopharyngeal carcinoma. Clin Radiol: 68: 683-689, 2013.

19. Matushansky I, Charytonowicz E, Mills J, Siddiqi S, Hricik T and Cordon-Cardo C: MFH classification: Differentiating undifferentiated pleomorphic sarcoma in the 21st century. Expert Rev Anticancer Ther 9: 1135-1144, 2009.

20. Al-Agha OM and Igbokwe AA: Malignant fibrous histiocytoma: Between the past and the present. Arch Pathol Lab Med 132: 1030-1035, 2008

21. Karki B, Xu YK, Wu YK and Zhang WW: Primary malignant fibrous histiocytoma of the abdominal cavity: CT findings and pathological correlation. World J Radiol 4: 151-158, 2012. 